\title{
Proyecto Internacional de Directores Exitosos de Escuelas de Nivel Secundaria
}

\author{
Eva Méndez Salcido \\ Escuela Normal Superior "Profr. José E. Medrano" \\ e.mendez@ensech.edu.mx
}

\begin{abstract}
Resumen
Este trabajo presenta los hallazgos preliminares del proyecto ISSPP por sus siglas en inglés (International Successful School Principalship Project). El reporte de investigación del caso CHI_01 es parte del proyecto de investigación internacional que tiene como objetivo documentar casos de directores de secundaria exitosos con el propósito de generar conocimiento que permita el desarrollo académico y profesional de directores de escuela actuales y futuros mediante la identificación de características y estrategias de administración y liderazgo exitoso en las escuelas secundarias.
\end{abstract}

Palabras clave

Directores, secundarias, teoría de la estructuración, agentes de cambio.

\section{Introducción y Contexto}

El trabajo presenta los hallazgos preliminares del estudio de caso CHIH_01 del proyecto ISSPP por sus siglas en inglés (International Successful School Principalship Project). El proyecto es parte de una investigación internacional donde participan instituciones de educación superior de 18 países. Tiene como objetivo documentar casos de directores de secundaria exitosos con el propósito de generar conocimiento que permita el desarrollo académico y profesional de directores de escuela actuales y futuros basándose en competencias identificadas.

El proyecto iniciado en el 2001 por Dr. Christopher Day de la Universidad de Nottingham en el Reino Unido, actualmente cuenta con 18 países participantes. En el caso de México docentes investigadores de 10 instituciones de educación superior han formado un grupo de investigación llamado Red ISSPP México. Estas instituciones son: Tecnológico de Monterrey, Universidad Pedagógica Nacional Unidad 261 de Hermosillo, Escuela Normal Superior de Jalisco, Escuela Normal Superior Oficial de Guanajuato, Escuela Normal de San Luis Potosí, Escuela Normal "Miguel F. Martínez" de Monterrey, Escuela Normal Superior de Yucatán, Escuela Normal Rural de Mactumactzá en Tuxtla Gutiérrez, Benemérita Escuela Normal Veracruzana "Enrique C. Rébsamen" y la Escuela Norma Superior "Profr. José E. Medrano R.".

La red ISSPP México consiguió en 2010 fondos de CONACyT para documentar 27 caso de directores exitosos en 9 ciudades de la república. En Chihuahua los investigadores Ildefonso Ruiz Benítez y Eva Méndez, profesores de la Escuela Norma Superior "Profr. José E. 
Medrano R." documentaron los casos de directores exitosos en la ciudad de Chihuahua. La selección de los directores fue mediante el cumplimiento de los criterios preestablecidos en el protocolo internacional. Cabe mencionar que dos de los 3 directores seleccionados pertenecen al subsistema federalizado y uno del subsistema estatal; dos de las 3 secundarias son modalidad general y una es modalidad técnica, lo que asegura diversos contextos escolares y formas de trabajo, enriqueciendo aún más los hallazgos en esta investigación.

El estudio de caso CHIH_01 documenta el caso del Profr. Carlos Avitia Aguilar que al momento del trabajo de campo contaba con dos años como director de los dos turnos de la escuela secundaria federal No.11, la cual se encuentra ubicada en la periferia de la zona urbana de la ciudad de Chihuahua.

\section{Marco teórico}

El objetivo del proyecto se centra en identificar y examinar las características y estrategias de una administración y liderazgo exitoso en las escuelas secundarias. Para tal efecto se toma como punto de partida al director como el actor que lleva a cabo la administración y guía los procesos en dichas instituciones.

Giddens (1995) menciona en su teoría de la estructuración la influencia que ejerce el actor en las prácticas sociales y cómo esas prácticas sociales obran en el actor generando un proceso dinámico, interdependiente $y$ situacional. Este proceso interdependiente desarrolla en el director la capacidad para producir y transformar circunstancias, convirtiéndolo en un agente de cambio.

Partiendo de la teoría de estructuración y de la idea de que el director es el agente de cambio que influye en las prácticas generadas dentro de la institución educativa se documenta el caso de un director de nivel secundaria para identificar las prácticas directivas que fomentan un mejoramiento en los resultados que se miden mediante los indicadores educativos.

\section{Metodología}

La metodología utilizada es de corte cualitativo, inductivo, utilizado para interpretar subjetivamente una realidad o situación. Es decir, se va de lo general a lo particular, explorando, describiendo $\mathrm{y}$ comprendiendo de manera inductiva (Bonilla \& Rodríguez, 2005). Se busca definir y explicar cualidades de un fenómeno, en este caso las características de un director exitoso y sus estrategias que contribuyen al éxito de la institución que dirige. En el enfoque cualitativo no se prueban hipótesis ya que estas se generan conforme avanza el proceso y se van modificando de acuerdo a los datos recopilados. Es muy importante que en los datos recopilados queden plasmados los puntos de vista y perspectivas de los participantes. Dado lo anterior y siguiendo los lineamientos de protocolo generado por la red internacional el trabajo de campo consistió en 3 entrevistas al director, entrevistas a padres de familia, a docentes y la realización de un grupo focal con alumnos de la institución, además de un cuestionario al director. El análisis de la información recabada se realizó mediante la triangulación de las diferentes fuentes de evidencia (Taylor y Bogdan, 1987): directores, profesores, padres y alumnos, organizando la información en las 5 categorías dadas por el protocolo antes mencionado.

La selección de los directores para el estudio de caso se basó en 6 criterios preestablecidos: 
1. Ser director de secundaria urbana de la ciudad de Chihuahua que sea exitoso(a).

2. Criterio de éxito: evidencia de mejoramiento en los indicadores que se usen en el estado desde la llegada del director(a).

3. De dos a cinco años en el puesto.

4. Que las autoridades lo consideren un buen director(a).

5. Que sus colegas lo consideren un buen director.

6. Que los padres y maestros lo consideren un buen director.

La selección inicial se realiza con los criterios 1, 2, 3, 4 y 5, el último criterio se verifica durante el trabajo de campo.

Las 5 categorías para la clasificación de la información son:

1. Los principales retos de la escuela (identificados desde la llegada del director).-

2. Estrategias que se utilizaron con éxito para enfrentar estos desafíos.

3. Cualidades percibidas $\mathrm{y}$ contribuciones del director y del equipo administrativo (perspectiva multi-actor)

4. Integración de alumnos procedentes de diferentes entornos sociales y culturales y la elevación de los niveles de logros.

5. Persistencia de problemas y nuevos desafíos: liderazgo propuesto y estrategias de administración

\section{Resultados}

Siguiendo el protocolo generado por la red internacional y buscando dar respuesta al objetivo del proyecto que se centra en identificar y examinar las características y estrategias de una administración y liderazgo que juegue un rol en el exitoso de las escuelas secundarias; para el caso CHIH_01 y a partir del trabajo de campo (entrevista: 3 al director, 7 a maestros, 6 a padres de familia $y$ grupos focales: 2 grupos de alumnos) y después de relacionar inductivamente la información recolectada, los resultados obtenidos en función de las 5 categorías dadas son: Retos principales: comunicación, infraestructura, atención/trato a alumnos, atención por parte de los padres de familia. Estrategias: presencia y comunicación. Características: estricto, accesible, involucrado. Logros: académico, infraestructura, reconocimiento. Retos persistentes: académico. 


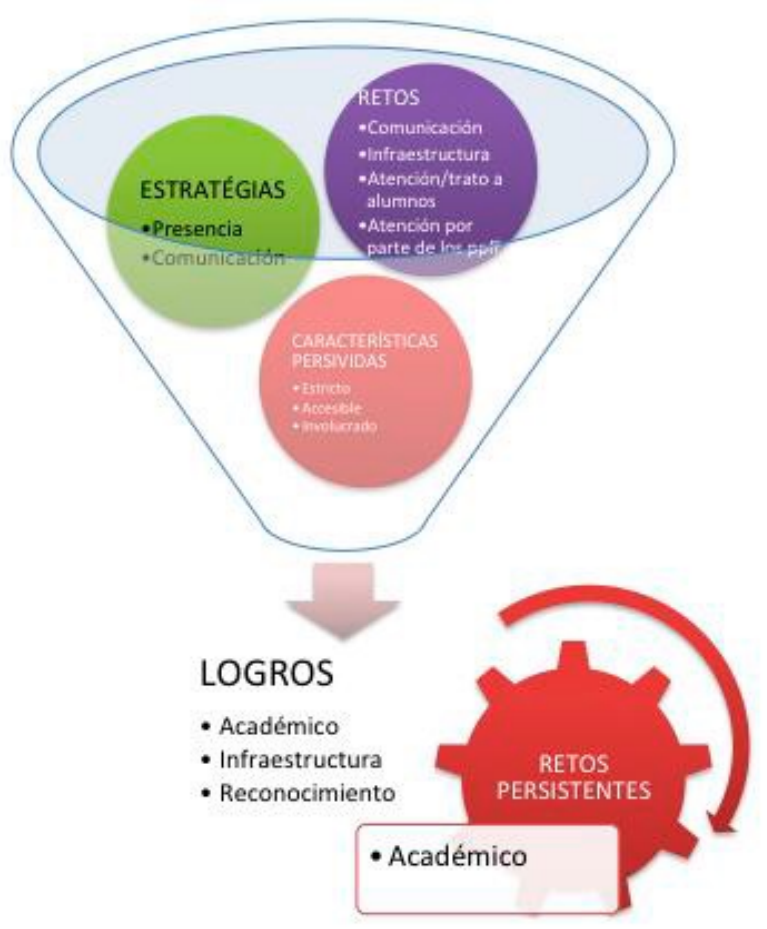

Figura 1.- Diagrama de categorías y subcategorías

A continuación se presentan las definiciones de las subcategorías identificadas en el caso CHIH_01, con respecto a las categorías las estrategias y características.

1. Los principales retos de la escuela (identificados desde la llegada del director).-

- Comunicación.- se refiere a la transmisión de información entre todos los actores, requerida para que los actores estén en conocimiento de los asuntos de interés.

- Infraestructura.- se refiere a las instalaciones físicas y equipamiento para el desarrollo de la enseñanza- aprendizaje.

- Atención / trato con alumnos.- Se refiere a que tan involucrados o al pendiente están los docentes de sus alumnos y sus necesidades tanto escolares como en otros ámbitos.

- Atención por parte de padres de familia.- Se refiere a que tan involucrados o al pendiente están los padres de familia de sus hijo y sus necesidades, tanto escolares como en otros ámbitos

2. Estrategias que se utilizaron con éxito para enfrentar estos desafíos.

- Presencia.- se refiere a la percepción de los diferentes actores de lo visible que es el director en todos los ámbitos y espacios de la institución.

- Comunicación.- se refiere a la transmisión de información entre todos los actores, requerida para que los actores estén en conocimiento de los asuntos de interés y se pueda detectar problemáticas u oportunidades de mejora en el servicio que se brinda. 
3. Cualidades percibidas y contribuciones del director y del equipo administrativo

- Estricto.- este concepto se maneja con relación a la forma de actuar del directo, cuando se necesita ser enérgico o serio lo es. Esto para hacer cumplir la normatividad y que las tareas se realicen.

- Accesible.- se refiere a que el profesor siempre se muestra abierto a atender a los padres de familia que necesiten ayuda o tengan alguna duda. Para él atenderlos es parte fundamental de su trabajo pues ellos son los beneficiarios del servicio de la escuela. Por lo que esta dispuesto a tomarse su tiempo para atenderlos.

- Involucrado.- se refiere a que el profesor es una persona que no cuida su imagen de autoridad como solamente Director, coordinador u organizador de las actividades, además de eso, él participa activamente en las diligencias y proyectos que se dan en la escuela.

4. Integración de alumnos procedentes de diferentes entornos sociales y culturales y la elevación de los niveles de logros. (Perspectiva multi-actor).

- Académico: al hablar de académico nos referimos al avance o no de los aprendizajes de los alumnos, el cual tiene como instrumento de medición las pruebas estandarizadas (ENLACE).

- Infraestructura: se refiere a los logros obtenidos en las instalaciones físicas y equipamiento para el desarrollo de la enseñanza- aprendizaje.

- Reconocimiento: se refiere a la opinión positiva que se tiene y se expresa de la institución educativa.

5. Persistencia de problemas y nuevos desafíos: liderazgo propuesto y estrategias de administración

- Académico: al hablar de académico nos referimos al avance en el aprendizaje por parte de los alumnos, el cual se mide mediante instrumentos estandarizados a nivel nacional (ENLACE).

En la figura 2: Categorías y subcategorías, se presentan las subcategorías encontradas en cada una de las categorías, indicando los actores que lo mencionaron, así como citas textuales tomadas de las transcripciones de las entrevistas y los grupos focales como evidencia de soporte.

En la categoría Principales Retos la subcategoría infraestructura es mencionada por los padres de familia, los maestros, alumnos y director; otra subcategoría es la comunicación mencionada por 3 actores, donde los maestros mencionan que se necesita una comunicación más activa y las otras dos subcategorías son atención/ trato con alumnos y atención por padres de familia que lo sugieren los maestros, padres de familia y el mismo director.

Como se puede observar en la gráfica en la categoría de estrategia una subcategoría es la presencia del director percibida por los padres de familia, los maestros y los alumnos, así como la mención del mismo director que intencionalmente toma como una práctica en su rutina diaria (Giddens, 1995) hacer sentir su presencia como parte de las actividades del director. Así mismo la comunicación es una subcategoría de la categoría estrategia mencionada por los 4 actores entrevistados.

En la categoría Cualidades se observa que las subcategorías accesible es mencionada por los padres de familia, los maestros y los alumnos; los alumnos y los maestros concuerdan en las subcategorías estricto; y los maestros y los padres de 
familia conciben al director como un actor involucrado. Por su parte el director menciona las tres subcategorías de la categoría Cualidades como elemento discursivo que busca de manera intencionada dar estructura a una dinámica institucional para generar y adoptar una rutina especifica en la escuela (Celis y Guatames, 2004).

En la categoría Integración de los alumnos y la elevación de los niveles de logro se observa que las subcategorías académico es mencionada por los 4 actores, que concuerdan que la prueba enlace ha sido un logro ya que tienen un alto nivel en ella; otra subcategoría señalada por todos los actores es el reconocimiento en ella se destaca las opiniones positivas de la institución motivo por el cual se da un carecimiento en la matrícula. Por ultimo infraestructura que es mencionada por los maestros, padres de familia y alumnos.

La última categoría es la Persistencia de problemas y nuevos desafíos y su subcategoría académico es mencionado por los padres de familia, maestros y el director los cuales tienen como objetivo elevar su nivel. 


\begin{tabular}{|c|c|c|c|}
\hline & $\begin{array}{c}\text { Principales cuestiones } 0 \\
\text { temas }\end{array}$ & $\begin{array}{c}\text { Evidencia de Soporte } \\
\text { (actores que lo mencionaron) }\end{array}$ & Citas \\
\hline \multirow{4}{*}{$\begin{array}{l}\text { 1. Los principales } \\
\text { retos de la escuela } \\
\text { (identificados desde } \\
\text { la llegada del } \\
\text { director).- }\end{array}$} & 1.-COMUNICACIÓN & MAESTROS, PPFF Y DIRECTOR & \begin{tabular}{|l|} 
1M, una comunicación más entre todos, que no se quede sólo en unos cuantos, sino que haya una comunicación \\
globalizada entre todos y viendo sobre todo las prioridades y necesidades de la secundaria \\
1P. si las planeaciones, es que el director revise pues algunas cosas que los maestros manejen con los alumnos, eso si me \\
gustaria saberlo, que revise u oiga pues que va a enseñar, que planeación trae y por qué, ..., si me gustaría que estuviera \\
más al pendiente de los programas que traen los profes \\
1D. a veces encuentra uno con algunas situaciones, eso un hace poquito ríspida la relación para con algunos, sobre todo \\
algunos que han dado motivos de, pues de acercarnos y tratar de cuestionar algunas prácticas que pues que \\
indudablemente se tienen que tratar, es por eso que la relación para con los profesores es buena hasta el margen donde a \\
veces no tocamos acciones laborales, ya cuando alguna de las dos partes, el director o el profesor dice ahl es que el \\
profesor me llamó la atención, o dice uno al es que este está fallando, es cuando se vuelve un poquito más frio,
\end{tabular} \\
\hline & 2. INFRAESTRUCTURA & $\begin{array}{l}\text { PPFF, MAESTROS, ALUMNOSY } \\
\text { DIRECTOR }\end{array}$ & $\begin{array}{l}\text { 1M..., que tuviéramos los edificios que nos faltan, o sea nos hace falta la dirección, la biblioteca } \\
\text { 1P. pues aquí hay mucho terreno verdad, se pudiera hacer más salones, no se para darle oportunidad a mas a más alumnos } \\
\text { a que entren y y tengan oportunidad de aprender aquí, } \\
\text { 1A...Por ejemplo hay un alumno que tiene discapacidades y que la escuela se adaptara para que se reconozca la escuela, } \\
\text { por si llega a venir otro estudiante asi que él no batallara, algo para las escaleras para que no se le vuelva tan pesado. } \\
\text { 1D nos atiendan las necesidades que hay fisica estructurales aquí en el edificio, que le inviertan a lo que es la tecnologia }\end{array}$ \\
\hline & $\begin{array}{l}\text { 3.-ATENCIÓN/TRATO CON } \\
\text { ALUMNOS }\end{array}$ & MAESTROS, PPFF Y DIRECTOR & $\begin{array}{l}\text { 1M siento que hay profesores que no, no se aplican o será que su materia se presta más ... } \\
\text { 1P. hay maestros que... no se preocupan por las criaturas, les va y les viene, a veces no llegan a clases, ... que estamos } \\
\text { comprometidos con nuestro trabajo, que la educación de sus hijos es lo más importante para nosotros } \\
\text { 10 mi desáfí es que reconozcan también una situación distinto, distinto de cada uno de los alumnos,... el desafío más } \\
\text { grande es que, tenemos que ir orientando a los maestros en tratar de entender a los alumnos }\end{array}$ \\
\hline & $\begin{array}{l}\text { ATENCIÓN POR PARTE DE } \\
\text { PADRES DE FAMILIA }\end{array}$ & MAESTROS, PPFF Y DIRECTOR & $\begin{array}{l}\text { 1M Que es absorbe mucho tiempo y si tenemos niños de padres que no trabajan o madres que están en el hogar pero ni se } \\
\text { notó su presencia estan poco participativas o no sé por qué. } \\
\text { 1P pero puedes por los padres hay muchos padres que no se responsabilizan por sus hijos y evitar lo acabamos de ver, } \\
\text { acabamos de tener una junta, estábamos en el salón } 17 \text { personas, póngale que llegaron } 20 \text { de } 40 \text { y tantos que son como } \\
\text { más de } 40 \text {... Pues yo creo un poco más de compromiso de los papás para que avancen más de lo que estaba, } \\
\text { 10 los padres de familia que que traen grandes problemas y que a veces son los propios hijos los que la llevan porque están } \\
\text { trabajando tantas horas para poder solventar tantos bienes o tantos servicios, pues la verdad es que es una gran } \\
\text { oroblemática. aoui tenemos a orandes alumnos muchos alumnos que están solos en su casa }\end{array}$ \\
\hline
\end{tabular}


RECIE. Revista Electrónica Científica de Investigación Educativa Vol. 1, núm. 2, enero-diciembre 2013, pp. 399-407.

\begin{tabular}{|c|c|c|c|}
\hline \multirow{2}{*}{$\begin{array}{l}\text { 2. Estrategias que se } \\
\text { utilizaron con éxito } \\
\text { para enfrentar estos } \\
\text { desafios. }\end{array}$} & PRESENCIA & $\begin{array}{l}\text { PPFF, MAESTROS, ALUMNOSY Y } \\
\text { DIRECTOR }\end{array}$ & $\begin{array}{l}\text { 2M Realmente con el profe Ávila es una persona muy, éste, activa es una persona, que está pendiente de que estemos } \\
\text { trabajando en ocasiones tenemos observaciones en el grupo, rondines. } \\
2 \mathrm{P} \text {.... casi siempre lo encontramos, ya ve que en la mayoria de las veces los directores pues no están... Incluso una vez } \\
\text { también que hubo aseo general no vinieron los papás que citaron, algunos cuantos y el director ahi andaba barriendo } \\
\text { 2A Que siempre se anda paseando en las clases y en el receso } \\
\text { 2D me gusta esperar al maestro en el salón de clases cuando esta llegando un poquito retrasado y no le digo nada, pero lo } \\
\text { espero ahi y el maestro entiende por que estoy yo ahi }\end{array}$ \\
\hline & COMUNICACIÓN & $\begin{array}{l}\text { PPFF, MAESTROS, ALUMNOSY } \\
\text { DIRECTOR }\end{array}$ & $\begin{array}{l}\text { 1M...cuando hay algún problema a algún un poquito más fuerte en que tal vez alguno tiene conocimiento de las situaciones } \\
\text { o de como manejar el problema puedes se habla con se le manda directamente con el subdirector o el director y ellos } \\
\text { atienden ese tipo de casos hasta el momento nos ha ido bien } \\
\text { 2a los profes son muy ablertos y podemos contar con ellos...2a Dándonos consejos } \\
\text { 2P. muchos padres de familia le ven de que no está tan cerrado, que si hay manera de expresarse cierlas inquietudes que } \\
\text { tenen a veces } \\
\text { 2D. cuando ellos (PPFF) anteponen alguna situación para con los maestros pues vamos con el maestro y decimos oye profe } \\
\text { se trata de esto..... a mi me gusta tener contacto con los padres de familia y ayudarlos en lo que yo pueda y los alumnos... } \\
\text { condescender con sus inquietudes y con los maestros...2D, damos espacio para que los maestros tengan sus academias } \\
\text { locales y que puedan intercambiar sus opiniones }\end{array}$ \\
\hline \multirow{3}{*}{$\begin{array}{l}\text { 3. Cualidades } \\
\text { percibidas y } \\
\text { contribuciones del } \\
\text { director y del equipo } \\
\text { administrativo } \\
\text { (perspectiva multi- } \\
\text { actor) }\end{array}$} & ESTRICTO & $\begin{array}{l}\text { MAESTROS, ALUMNOSY } \\
\text { DIRECTOR }\end{array}$ & $\begin{array}{l}\text { 3D hay momentos en el que somos muy ecuànimes, hay momentos estamos muy serios, demostramos, cierta inconformidad } \\
\text { a nuestra manera } \\
\text { 3M el sabe donde, como trabajar con flexibilidad y cuando trabajar con un poquito de energia, estricto } \\
\text { 3A El direclor, pues me cae bien es muy divertido, es muy disciplinado y sabe hacer como hacer la escuela educada, estricto }\end{array}$ \\
\hline & ACCESIBLE & $\begin{array}{l}\text { PPFF, MAESTROS, ALUMNOS Y } \\
\text { DIRECTOR }\end{array}$ & $\begin{array}{l}\text { 3M muy accesible a la mayoría si no es que todos los atiende } \\
\text { 3P siempre esta dispuesto a atender a los padres de familia aunque haga uno fila siempre está dispuesto a atenderlo a la } \\
\text { hora que sea } \\
\text { 3A Que es una persona muy abierta }\end{array}$ \\
\hline & INVOLUCRADO & PPFF, MAESTROS Y DIRECTOR & $\begin{array}{l}\text { 3D yo también puedo levantar una basura cualquiera, para cooperar y de esa manera encarrilarlos } \\
\text { 3M Yo le digo se involucra demasiado profe, porque si se trata de barrer en la ayuda, si se trata de repartir boletas } \\
\text { 3P. está al pendiente, de lo que estân sucediendo afuera verdad entre los jovene }\end{array}$ \\
\hline \multirow{3}{*}{$\begin{array}{l}\text { 4. Integración de } \\
\text { alumnos procedentes } \\
\text { de diferentes } \\
\text { entornos sociales y } \\
\text { culturales y la } \\
\text { elevación de los } \\
\text { niveles de legres. }\end{array}$} & ACADÉMICO & $\begin{array}{l}\text { PPFF, MAESTROS, ALUMNOSY } \\
\text { DIRECTOR }\end{array}$ & $\begin{array}{l}\text { 4D. Hay resultados en ENLACE. } \\
\text { 4M ENLACE, pues poco a poquito vamos subiendo... Ha subido el nivel académico } \\
\text { 4P La prueba ENLACE es un gran logro verdad } \\
\text { 4A Que tiene alto nivel académico }\end{array}$ \\
\hline & INFRAESTRUCTURA & PPFF, MAESTROS, ALUMNOS & $\begin{array}{l}\text { 4M Ha atendido las necesidades de la infraestructura } \\
\text { 4P Han avanzado mucho en trabajos de de ampliación verdad } \\
\text { 4A Que están haciendo la cafetería, plantar árboles y todo eso }\end{array}$ \\
\hline & RECONOCIMIENTO & $\begin{array}{l}\text { PPFF, MAESTROS, ALUMNOEY Y } \\
\text { DIRECTOR }\end{array}$ & $\begin{array}{l}\text { 4P La demanda ha crecido bastante } \\
\text { 4M Me parece muy buena escuela... Yo me fijé en esta escuela por los comentarios que habia escuchado } \\
\text { 4A Que cota os una de las mojorca accundarias que hay on cl oatado } \\
\text { 1D. El reconocimiento ese es el orgullo el orgullo que tenemos aqui los logros más grandes son los que vienen de allá para } \\
\text { acá, y si nuestras autoridades también reconocen por que también hemos tenido buenos }\end{array}$ \\
\hline $\begin{array}{l}\text { 5. Persistencia de } \\
\text { problemas y } \\
\text { nuevos desafios: } \\
\text { liderazgo propuesto } \\
\text { y estrategias de } \\
\text { administración. }\end{array}$ & ACADÉMICO & MAESTROS, PPFF Y DIRECTOR & 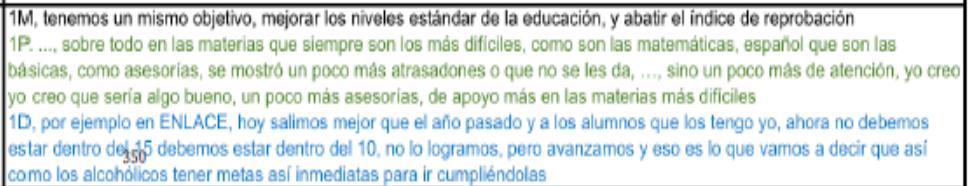 \\
\hline
\end{tabular}




\section{Conclusiones e implicaciones}

Este trabajo de investigación sobre la documentación del caso CHI_01 director exitoso de escuela secundaria de Chihuahua nos describe las cualidades: estricto, accesible e involucrado, y las principales estrategias: presencia $\mathrm{y}$ comunicación, que el director en cuestión pone en práctica al administrar y liderar con éxito la escuela secundarias que preside y dar frente a los principales retos, reconociendo que el reto persistente es el nivel académico aun y cuando se reconoce cierto logro al respecto.

Estos resultados nos dan un punto de partida para generar formas de desarrollo profesional que permita a directores y/o candidatos a una dirección desenvolverse como administradores educativos exitosos.

Cabe señalar que si bien a nivel internacional existen más de 100 casos de directores exitosos documentados, parte de las implicaciones de este proyecto es ampliar la documentación de casos en la región del estado de Chihuahua que permitan visualizar de manera más profunda los atributos con los que debe contar un director de escuela secundaria para asegurar el éxito de la labor educativa.

\section{Referentes}

Bonilla, E. \& Rodríguez, P. (2005). Más allá del dilema de los métodos. Colombia: Editorial Nomos S.A.

Celis, J. E. y Guatame, P. C., (2004). La institución educativa y la teoría de la estructuración de Anthony Giddens. Revista Iberoamericana de Educación No. 33/9

Giddens, A. (1995). La Constitución de la Sociedad. Bases para teoría de la estructuración. Buenos Aires. Amorrortu.

Taylor, S. J., \& Bogdan, R. (1987). Introducción a los métodos cualitativos de investigación. (J. Piatigorsky, Trad.) Barcelona, España: Paidós. 
RECIE. Revista Electrónica Científica de Investigación Educativa Vol. 1, núm. 2, enero-diciembre 2013, pp. 399-407. 